\title{
ISS-Lyapunov Functions for Discontinuous Discrete-Time Systems
}

\author{
Lars Grüne and Christopher M. Kellett
}

\begin{abstract}
Input-to-State Stability (ISS) and the ISS-Lyapunov function are useful tools for the analysis and design of nonlinear systems. Motivated by the fact that many feedback control laws, such as model predictive or event-based control, lead to discontinuous discrete-time dynamics, we investigate ISSLyapunov functions for such systems. ISS-Lyapunov functions were originally introduced in a so-called implication-form and, in many cases, this has been shown to be equivalent to an ISS-Lyapunov function of dissipative form. However, for discontinuous dynamics, we demonstrate via an example that this equivalence no longer holds. We therefore propose a stronger implication-form ISS-Lyapunov function and provide a complete characterization of ISS-Lyapunov functions for discrete-time systems with discontinuous dynamics.
\end{abstract}

\section{Index Terms}

Input-to-State Stability (ISS), Lyapunov Methods, Discrete-Time Systems

\section{INTRODUCTION}

Originally formulated in continuous time, the notion of input-to-state stability (ISS) introduced by Sontag in [20] was soon adapted to discrete time systems. In this paper, we consider ISSLyapunov functions, first introduced in [23], for discrete-time nonlinear systems given by

$$
x^{+}=f(x, w)
$$

L. Grüne is with the Mathematisches Institut, Universität Bayreuth, 95440 Bayreuth, Germany, e-mail: lars.grueneduni-bayreuth.de.

C. M. Kellett is with the School of Electrical Engineering and Computer Science at the University of Newcastle, Callaghan, New South Wales 2308, Australia, e-mail: Chris.Kellett@newcastle.edu.au. 
where $x \in \mathbb{R}^{n}, w \in \mathbb{R}^{m}$, and $f: \mathbb{R}^{n} \times \mathbb{R}^{m} \rightarrow \mathbb{R}^{n}$. We take as inputs sequences with values in $\mathbb{R}^{m}$ and we denote this space by $\mathcal{W}$. We denote solutions of (1) by $\phi: \mathbb{Z}_{\geq 0} \times \mathbb{R}^{n} \times \mathcal{W} \rightarrow \mathbb{R}^{n}$.

Many continuous-time ISS results carry over to the discrete-time setting if the discrete time dynamics are continuous, see [7]. However, this statement is no longer true when discontinuous dynamics are considered and in this paper we will not impose any regularity assumptions on $f(\cdot, \cdot)$. Besides the fact that certain models naturally lead to discontinuous dynamics, our main motivation for considering discontinuous $f$ are controller design techniques that lead to discontinuous dynamics. Indeed, even if the controlled dynamics $x^{+}=g(x, u, w)$ with control input $u \in U$ is continuous, the use of a discontinuous controller $u: \mathbb{R}^{n} \rightarrow U$ leads to a discontinuous closed loop system $x^{+}=g(x, u(x), w)=: f(x, w)$ of the form (1). Among modern controller design techniques, optimization based techniques such as model predictive control (MPC) naturally lead to discontinuous feedback laws and, in the presence of state constraints, even the corresponding Lyapunov function is typically discontinuous, cf. [3], [18] or [5, Sections 8.5-8.9]. Similarly, quantized [4], [16] or event-based [2], [14] feedback laws naturally lead to discontinuous closed loop dynamics.

It was observed before that additional assumptions are required in order to make the usual ISS-Lyapunov function arguments work for discontinuous discrete time systems, see, e.g., [6, Assumptions 7 and 8]. Also, it is known that discontinuities may affect the usual inherent robustness properties of, e.g., asymptotic [11] or exponential stability [13]. It is the goal of this paper to give a comprehensive and rigorous collection of results on ISS-Lyapunov functions for discontinuous systems. Particularly, we present necessary and sufficient Lyapunov function characterizations of ISS, discuss the equivalence of different types of decay estimates for ISS Lyapunov functions, and introduce a stronger variant of an implication-form Lyapunov function that is demonstrated to be better suited to the discontinuous setting.

The paper is organized as follows. In Section II we recall the definitions of input-to-state stability (ISS) and dissipative-form ISS-Lyapunov functions and discuss the relation between these concepts as well as different decay properties of the Lyapunov functions in the discontinuous setting. In Section III we recall the standard definition of an implication-form ISS-Lyapunov function and show that in the presence of discontinuities additional conditions are needed in order to conclude ISS from the existence of these Lyapunov functions. In Section IV we present and analyze our new stronger implication-form ISS-Lyapunov function. We show that the existence of 
such a function is indeed equivalent to the ISS property for discontinuous systems and illustrate the usefulness of this concept by proving two propositions yielding sufficient conditions for ISS. Conclusions are presented in Section V and proofs of the main results can be found in Section VI.

\section{ISS AND DISSIPATIVE-FORM ISS-LYAPUNOV FUNCTIONS}

In the sequel, we will denote the class of continuous positive definite functions $\rho: \mathbb{R}_{\geq 0} \rightarrow \mathbb{R}_{\geq 0}$ by $\mathcal{P}$. We will also make use of the standard function classes $\mathcal{K}, \mathcal{K}_{\infty}, \mathcal{L}$, and $\mathcal{K} \mathcal{L}$ (see [9]).

Definition 2.1: The system (1) is input-to-state stable (ISS) if there exist $\beta \in \mathcal{K} \mathcal{L}, \gamma \in \mathcal{K}$ such that

$$
|\phi(k, x, w)| \leq \max \left\{\beta(|x|, k), \max _{i \in \mathbb{Z}_{[0, k-1]}} \gamma(|w(i)|)\right\}
$$

for all $x \in \mathbb{R}^{n}, w \in \mathcal{W}$, and $k \in \mathbb{Z}_{\geq 0}$.

Definition 2.2: A dissipative ISS-Lyapunov function for (1) is a function $V: \mathbb{R}^{n} \rightarrow \mathbb{R}_{\geq 0}$ such that there exist $\alpha_{1}, \alpha_{2}, \alpha \in \mathcal{K}_{\infty}$ and $\sigma \in \mathcal{K}$ so that, for all $x \in \mathbb{R}^{n}$ and $w \in \mathbb{R}^{m}$

$$
\begin{gathered}
\alpha_{1}(|x|) \leq V(x) \leq \alpha_{2}(|x|) \\
V(f(x, w))-V(x) \leq-\alpha(|x|)+\sigma(|w|) .
\end{gathered}
$$

A dissipative ISS-Lyapunov function is called exponential if there exists $\lambda \in(0,1)$ such that (4) can be written in the form

$$
V(f(x, w)) \leq \lambda V(x)+\sigma(|w|) .
$$

An implicit constraint in (4) is that $\alpha(|x|) \leq V(x)$ for all $x \in \mathbb{R}^{n}$.

Despite the lack of any regularity assumptions on either the system dynamics (1) or the ISS-Lyapunov function (3)-(4), we may prove the following theorem.

Theorem 2.3: The following statements are equivalent.

(i) The system (1) is ISS;

(ii) There exists a dissipative ISS-Lyapunov function $V$;

(iii) For any $\lambda \in(0,1)$ there exists an exponential dissipative ISS-Lyapunov function $\widehat{V}$.

Proof: “(iii) $\Rightarrow$ (ii)" follows immediately from Definition 2.2 and "(ii) $\Rightarrow$ (i)" is [7, Lemma 3.5], noting that the continuity assumptions on the dynamics and the Lyapunov function imposed in 
[7] play no part in the proof of [7, Lemma 3.5]. The proof of "(i) $\Rightarrow$ (iii)" can be found in Section VI-A.

We note that the existence of an exponentially decaying Lyapunov function does not imply anything about the decay rate of the system trajectories.

Remark 2.4: The equivalence (ii) $\Leftrightarrow$ (iii) in Theorem 2.3 provides a discrete time and discontinuous version of [19, Proposition 8]. We will show in Remark 4.5 that the Lyapunov function $\widehat{V}$ in (iii) can be explicitly derived from $V$ in (ii) as $\widehat{V}=\hat{\alpha}(V)$ for some $\hat{\alpha} \in \mathcal{K}_{\infty}$.

The implication "(ii) $\Rightarrow$ (i)" in Theorem 2.3 may not hold when relaxing the assumption $\alpha \in \mathcal{K}_{\infty}$ in Definition 2.2. We end this section by observing that this assumption can be weakened to $\alpha \in \mathcal{K}$ if an additional compatibility condition between $\alpha$ and $\sigma$ holds.

Proposition 2.5: Suppose $V: \mathbb{R}^{n} \rightarrow \mathbb{R}_{\geq 0}, \alpha_{1}, \alpha_{2} \in \mathcal{K}_{\infty}$ and $\alpha, \sigma \in \mathcal{K}$ satisfy (3) and (4). If $\sup \alpha>\sup \sigma$, then (1) is ISS.

This follows from results on changing supply functions for ISS systems presented in [22] for continuous time and in [17] for discrete-time. We provide the proof in Section VI-B.

\section{IMPLICATION-FORM ISS-LYAPUNOV FUNCTIONS}

As an alternate to the dissipative decrease condition (4), the following "implication-form" ISS-Lyapunov function has frequently been used in the literature:

Definition 3.1: An implication-form ISS-Lyapunov function for (1) is a function $V: \mathbb{R}^{n} \rightarrow \mathbb{R}_{\geq 0}$ such that there exist $\alpha_{1}, \alpha_{2} \in \mathcal{K}_{\infty}, \chi \in \mathcal{K}$, and $\rho \in \mathcal{P}$ so that, for all $x \in \mathbb{R}^{n}$ and $w \in \mathbb{R}^{m}$, (3) holds and

$$
|x| \geq \chi(|w|) \quad \Rightarrow \quad V(f(x, w))-V(x) \leq-\rho(|x|) .
$$

As before, there is an implicit constraint that $\rho(|x|) \leq V(x)$.

Having a merely positive definite decrease rate $\rho$ as in (6) is not necessarily convenient for calculations. In many cases, rather than (6) it is useful to have the following class- $\mathcal{K}_{\infty}$ decrease rate: let $V: \mathbb{R}^{n} \rightarrow \mathbb{R}_{\geq 0}$ and $\alpha_{1}, \alpha_{2}, \bar{\alpha} \in \mathcal{K}_{\infty}$ and $\chi \in \mathcal{K}$ satisfy (3) and

$$
|x| \geq \chi(|w|) \quad \Rightarrow \quad V(f(x, w))-V(x) \leq-\bar{\alpha}(|x|) .
$$


A further potentially useful refinement is the following exponential decrease rate: let $V$ : $\mathbb{R}^{n} \rightarrow \mathbb{R}_{\geq 0}$ and $\alpha_{1}, \alpha_{2} \in \mathcal{K}_{\infty}, \hat{\lambda} \in(0,1)$, and $\chi \in \mathcal{K}$ satisfy (3) and

$$
|x| \geq \chi(|w|) \quad \Rightarrow \quad V(f(x, w)) \leq \hat{\lambda} V(x)
$$

We can demonstrate the following relationship between these three implication-form ISSLyapunov functions.

Proposition 3.2: The following are equivalent:

(i) There exist a $\rho \in \mathcal{P}$ and an implication-form ISS-Lyapunov function $V$ satisfying (6);

(ii) There exist an $\bar{\alpha} \in \mathcal{K}_{\infty}$ and an implication-form ISS-Lyapunov function $\widehat{V}$ satisfying (7);

(iii) For any given $\hat{\lambda} \in(0,1)$ there exists an implication-form ISS-Lyapunov function $\widetilde{V}$ satisfying (8).

Moreover, for $V$ satisfying (i) there exist $\hat{\alpha}, \tilde{\alpha} \in \mathcal{K}_{\infty}$ such that $\widehat{V}$ in (ii) and $\widetilde{V}$ in (iii) can be written in the form $\widehat{V}=\hat{\alpha}(V)$ and $\widetilde{V}=\tilde{\alpha}(V)$.

The equivalence of (i) and (ii) was stated in [7, Remark 3.3] and the proof follows as in [8, Lemma 2.8]. The equivalence of (ii) and (iii) follows an argument in the proof of [10, Theorem 6]. The complete proof is provided in Section VI-C.

Theorem 2.3 states that the existence of a dissipative ISS-Lyapunov function implies ISS. By contrast, the following example shows that an implication-form ISS-Lyapunov function does not necessarily imply ISS.

Example 3.3: Consider the system

$$
x^{+}=f(x, w)=\nu(w) \kappa(x)
$$

where

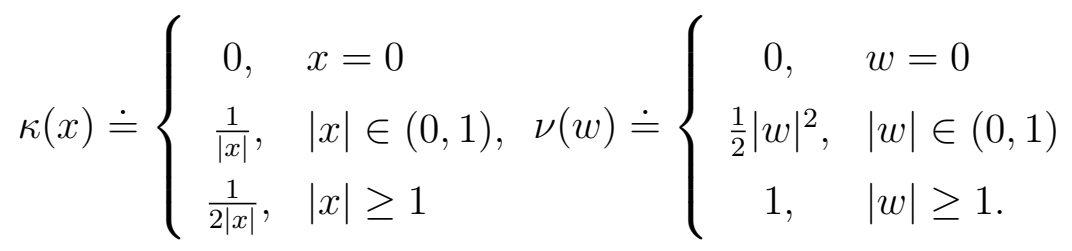

Take $V(x) \doteq|x|$ so that both the upper and lower bounds of (3) can be trivially taken as $|x|$. We observe that if $|x| \geq|w|$ then for $|x| \in(0,1)$

$$
|f(x, w)|=\frac{|w|^{2}}{2|x|} \leq \frac{|x|^{2}}{2|x|}=\frac{|x|}{2}
$$

while for $|x| \geq 1$ we have $|f(x, w)|=\nu(w) \frac{1}{2|x|} \leq \frac{1}{2|x|} \leq \frac{|x|}{2}$. 
Therefore, with $\alpha(s) \doteq \frac{1}{2} s$ we see that

$$
|x| \geq|w| \Rightarrow V(f(x, w))-V(x) \leq-\alpha(|x|) .
$$

However, it is straightforward to see that the system (9) is not ISS. Take $w \equiv 1$ and any initial condition $x \in(0,1)$. Then $\phi(2 k+1, x)=2^{2 k} / x$ for all $k \in \mathbb{Z}_{\geq 0}$. In other words, every other time step the solution increases so that the ISS estimate (2) can never be satisfied.

This example shows that the existence of an implication-form ISS-Lyapunov function does not imply the existence of a dissipative ISS-Lyapunov functions, since according to Theorem 2.3 the latter would be equivalent to the system being ISS. This motivates the need for additional conditions which enable us to construct dissipative ISS-Lyapunov functions from those in implication-form. The following two propositions provide sufficient conditions for this purpose.

Proposition 3.4: Assume that system (1) satisfies the ISS-estimate (2) and that $V: \mathbb{R}^{n} \rightarrow \mathbb{R}_{\geq 0}$, $\alpha_{1}, \alpha_{2}, \bar{\alpha} \in \mathcal{K}_{\infty}$ and $\chi \in \mathcal{K}$ satisfy (3) and (7). Then $V$ also satisfies (4) with $\alpha \doteq \min \left\{\bar{\alpha}, \alpha_{1}\right\}$ and $\sigma(s) \doteq \alpha_{2}(\beta(\chi(s), 1)+\gamma(s))$ for all $s \in \mathbb{R}_{\geq 0}$.

The proof makes explicit use of the ISS-estimate to ensure that the potential increase in the ISS-Lyapunov function is bounded for states below the level set defined by $\chi(|w|)$. This is similar to the final argument at the end of the proof of "(i) $\Rightarrow$ (iii)" in Theorem 2.3 and we thus omit the details.

The assumption of ISS immediately yields a dissipative form ISS-Lyapunov function (by Theorem 2.3) and consequently, the only novelty of Proposition 3.4 is that for ISS systems any implication-form ISS-Lyapunov function is also a dissipative ISS-Lyapunov function. Note that Proposition 3.4 does not rely on the regularity of either the system dynamics or the ISS-Lyapunov functions.

Rather than assuming that system (1) is ISS to show the result of Proposition 3.4, we may assume continuity of both the ISS-Lyapunov function and the system dynamics. This was already stated in [7, Remark 3.3] without proof.

Proposition 3.5: Assume $f(\cdot, \cdot)$ is continuous. If there exist a continuous function $V: \mathbb{R}^{n} \rightarrow$ $\mathbb{R}_{\geq 0}$ and functions $\alpha_{1}, \alpha_{2} \in \mathcal{K}_{\infty}$ and $\bar{\alpha}, \chi \in \mathcal{K}$ satisfying (3) and (7), then $V$ satisfies (4) with $\alpha \doteq \bar{\alpha}$ and

$$
\sigma(r) \doteq \max \left\{\begin{array}{c|l}
V(f(x, w))-V(x) & |w| \leq r \\
+\alpha \circ \chi(|w|) & |x| \leq \chi(r)
\end{array}\right\}
$$


Proof: The proof follows the same argument as in [23, Remark 2.4]. That the maximum in (10) is well-defined follows from the fact that the function being maximized is a continuous function by the assumptions on $V(\cdot)$ and $f(\cdot, \cdot)$ and the fact that the domain being maximized over is compact. It is then straightforward to see that

$$
\sigma(|w|) \geq V(f(x, w))-V(x)+\alpha(|x|)
$$

yielding the desired result.

The converse of Proposition 3.5 holds without any continuity properties:

Proposition 3.6: If there exist a function $V: \mathbb{R}^{n} \rightarrow \mathbb{R}_{\geq 0}$ and functions $\alpha_{1}, \alpha_{2}, \alpha \in \mathcal{K}_{\infty}$ and $\sigma \in \mathcal{K}$ satisfying (3) and (4) then $V$ satisfies (7) with $\chi \doteq \alpha^{-1} \circ 2 \sigma \in \mathcal{K}$ and $\bar{\alpha}=\frac{1}{2} \alpha \in \mathcal{K}_{\infty}$.

Proof: We rewrite (4) as $V(f(x, w))-V(x) \leq-\alpha(|x|) / 2-\alpha(|x|) / 2+\sigma(|w|)$. Then, with $\chi \doteq \alpha^{-1} \circ 2 \sigma \in \mathcal{K}$ and $\bar{\alpha} \doteq \frac{1}{2} \alpha \in \mathcal{K}_{\infty}$ we immediately see $|x| \geq \chi(|w|) \Rightarrow V(f(x, w))-V(x) \leq$ $-\frac{1}{2} \alpha(|x|)=\bar{\alpha}(|x|)$.

Remark 3.7: We observe that we can trade off the decrease rate, $\bar{\alpha}$ and the input-dependent level set defined by $\chi$. In particular, for any $\rho \in \mathcal{P}$ and $\varphi \in \mathcal{K}_{\infty}$ such that $\rho(s)+\varphi(s) \leq \alpha(s)$, for all $s \in \mathbb{R}_{\geq 0}$ we see that $V$ satisfies (6) with $\hat{\rho} \doteq \rho$ and $\chi \doteq \varphi^{-1} \circ \sigma$.

We note that, as an interim step in the proof of "(i) $\Rightarrow$ (iii)" in Theorem 2.3, we demonstrate that ISS implies the existence of an implication-form ISS-Lyapunov function satisfying (3) and (8). However, the above example demonstrates that the converse is not true. Hence, this indicates that neither the implication-form of (8), nor the equivalent forms demonstrated by Proposition 3.2, are useful when one allows discontinuous system dynamics since (3) and (7) do not imply ISS of (1). This motivates a new definition for implication-form ISS-Lyapunov functions in the following section.

\section{AN ALTERNATIVE IMPLICATION-FORM ISS-LYAPUNOV FUNCTION}

As we have seen, in the discontinuous setting the existence of an ISS-Lyapunov function in the implication-form (3), (7) does not imply ISS and is not equivalent to the existence of an ISS-Lyapunov function in dissipation form (3), (4). We propose the following stronger alternative to the implication (6) which fixes these problems.

Definition 4.1: A strong implication-form ISS-Lyapunov function for (1) is a function $V$ : $\mathbb{R}^{n} \rightarrow \mathbb{R}_{\geq 0}$ such that there exist functions $\alpha_{1}, \alpha_{2} \in \mathcal{K}_{\infty}, \hat{\varphi}, \chi \in \mathcal{K}$, and $\hat{\rho} \in \mathcal{P}$ so that, for all 
$x \in \mathbb{R}^{n}$ and $w \in \mathbb{R}^{m}, V$ satisfies (3) and

$$
\begin{aligned}
& |x| \geq \chi(|w|) \quad \Rightarrow \quad V(f(x, w))-V(x) \leq-\hat{\rho}(|x|) \\
& |x|<\chi(|w|) \quad \Rightarrow \quad V(f(x, w)) \leq \hat{\varphi}(|w|) .
\end{aligned}
$$

This definition is motivated by the ISS Lyapunov functions in implication-form in continuous time, which will always satisfy the second implication on time intervals on which $w$ is constant.

Remark 4.2: Regional or local ISS on some subset $\Theta \subset \mathbb{R}^{n}$ is of particular importance in MPC [15]. To extend the results of this paper to the regional ISS setting, in addition to the modifications required as described in [15], $\hat{\varphi} \in \mathcal{K}$ of Definition 4.1 would need to ensure that possible jumps from the set $|x|<\chi(|w|)$ do not result in trajectories outside of the region of interest, $\Theta$.

Remark 4.3: Proposition 3.2 applies analogously to the strong implication-form, cf. its proof in Section VI-C. This means that by rescaling $V$ to $\hat{\alpha}(V)$ and $\tilde{\alpha}(V)$ we can pass from (11) to (7) or (8), respectively.

In the particular case of a $V$ satisfying (3), (8), and (12) we call $V$ an exponential strong implication-form ISS-Lyapunov function.

The next theorem shows that the concept of strong implication-form ISS-Lyapunov function overcomes the gap observed between a dissipative ISS-Lyapunov function and the classical implication-form ISS-Lyapunov function from Definition 3.1 when considering discontinuous systems and ISS-Lyapunov functions.

Theorem 4.4: The following statements are equivalent.

(i) The system (1) is ISS;

(ii) There exists a strong implication-form ISS-Lyapunov function $V$;

(iii) For any $\lambda \in(0,1)$ there exists an exponential strong implication-form ISS-Lyapunov function $\widehat{V}$.

Moreover,

(a) $V$ is a strong implication-form ISS-Lyapunov function satisfying (7) and (12) if and only if $V$ is a dissipative ISS-Lyapunov function;

(b) $V$ is an exponential strong implication-form ISS-Lyapunov function if and only if $V$ is an exponential dissipative ISS-Lyapunov. 
Proof: We first prove (a) and (b).

(a), " $\Rightarrow$ ": Let $V$ together with functions $\bar{\alpha} \in \mathcal{K}_{\infty}$ and $\chi, \hat{\varphi} \in \mathcal{K}$ satisfy (7) and (12). We claim that $V$ satisfies (4) with $\alpha \doteq \min \left\{\bar{\alpha}, \alpha_{1}\right\}$ and $\sigma=\hat{\varphi}$ : If $|x| \geq \chi(|w|)$ then we get $V(f(x, w))-V(x) \leq-\bar{\alpha}(|x|) \leq-\alpha(|x|)+\sigma(|w|)$. In case $|x|<\chi(|w|)$, using (3) we obtain $V(f(x, w))-V(x) \leq \hat{\varphi}(|w|)-\alpha_{1}(|x|) \leq-\alpha(|x|)+\sigma(|w|)$.

(a), " $\Leftarrow$ ": Let $V$ together with functions $\alpha \in \mathcal{K}_{\infty}$ and $\sigma \in \mathcal{K}$ satisfy (4). Then $V$ also satisfies (7) with $\bar{\alpha} \doteq \alpha / 2, \chi \doteq \alpha^{-1} \circ 2 \sigma$ and (12) with $\hat{\varphi} \doteq \gamma \circ \chi+\sigma$, where $\gamma \in \mathcal{K}_{\infty}$ is arbitrary with $\gamma \geq \alpha_{2}-\alpha$. To see this, we rewrite (4) as $V(f(x, w))-V(x) \leq-\alpha(|x|) / 2-\alpha(|x|) / 2+\sigma(|w|)$. Then, with $\chi \doteq \alpha^{-1} \circ 2 \sigma \in \mathcal{K}$ and $\bar{\alpha} \doteq \frac{1}{2} \alpha \in \mathcal{K}_{\infty}$ we see that $|x| \geq \chi(|w|)$ implies $V(f(x, w))-$ $V(x) \leq-\alpha(|x|) / 2=-\bar{\alpha}(|x|)$ and $|x|<\chi(|w|)$ implies

$$
\begin{aligned}
V(f(x, w)) & \leq V(x)-\alpha(|x|)+\sigma(|w|) \\
& \leq \alpha_{2}(|x|)-\alpha(|x|)+\sigma(|w|) \leq \gamma(|x|)+\sigma(|w|) \\
& \leq \gamma(\chi(|w|))+\sigma(|w|)=\hat{\varphi}(|w|) .
\end{aligned}
$$

(b), " $\Rightarrow$ ": We prove that if $V$ together with $\lambda \in(0,1)$ and $\sigma \in \mathcal{K}$ satisfies (5), then $V$ also satisfies (12) and (8) with $\hat{\lambda} \doteq \lambda+\varepsilon$ satisfying $\lambda+\varepsilon<1, \chi \doteq \alpha_{1}^{-1}\left(\frac{1}{\varepsilon} \sigma\right)$, and $\hat{\varphi} \doteq \lambda \alpha_{2} \circ \chi+\sigma$. To this end, we may then rewrite (5) as

$$
\begin{aligned}
V(f(x, w)) & \leq(\lambda+\varepsilon) V(x)-\varepsilon V(x)+\sigma(|w|) \\
& \leq \hat{\lambda} V(x)-\varepsilon \alpha_{1}(|x|)+\sigma(|w|)
\end{aligned}
$$

which yields the implication (8). The implication (12) follows from the upper bound on $V$ and the condition $|x|<\chi(|w|)$ as $V(f(x, w)) \leq \lambda \alpha_{2}(|x|)+\sigma(|w|) \leq \lambda \alpha_{2} \circ \chi(|w|)+\sigma(|w|)$.

(b), " $\Leftarrow$ ": A straightforward calculation shows that if $V$ satisfies (12) and (8), then $V$ also satisfies (5) with $\sigma \doteq \hat{\varphi}$.

Now, the equivalences (i) $\Leftrightarrow$ (ii) $\Leftrightarrow$ (iii) follow immediately from (a), (b) and Theorem 2.3.

Remark 4.5: Theorem 4.4 reveals that we can explicitly choose $\widehat{V}$ in Theorem 2.3 in the form $\widehat{V}=\hat{\alpha}(V)$ for some $\hat{\alpha} \in \mathcal{K}_{\infty}$. Indeed, by Theorem 4.4(a) the dissipative ISS-Lyapunov function $V$ in Theorem 2.3 is also a strong implication-form ISS-Lyapunov function. Proposition 3.2 and Remark 4.3 then show that rescaling $V$ with $\tilde{\alpha} \in \mathcal{K}_{\infty}$ yields an exponential strong implicationform ISS-Lyapunov function which, by Theorem 4.4(b), is also an exponential ISS-Lyapunov function in dissipative form. 
Theorem 4.4 can be used in order to pass from the (weak) implication-form (7) to the dissipation form (4) under a weaker continuity assumption than in Proposition 3.5. Particularly, we only require continuity of $f$ at $w=0$ (uniformly in $x$ ).

Proposition 4.6: Let $V$ be a function satisfying (3) and (7) for appropriate $\alpha_{1}, \alpha_{2}, \bar{\alpha} \in \mathcal{K}_{\infty}$ and $\chi \in \mathcal{K}$. Assume that $f$ is continuous in $w=0$ uniformly in $x$ in the following sense:

For each $r>0$ there is $\gamma_{r} \in \mathcal{K}_{\infty}$ such that for all $|x| \leq r,|w| \leq r$ the map $f$ satisfies the inequality $|f(x, w)-f(x, 0)| \leq \gamma_{r}(|w|)$.

Then there exists $\hat{\varphi} \in \mathcal{K}$ so that $V$ satisfies (12) and thus also (4).

Proof: First, consider $w \equiv 0$. Then we observe that

$$
\begin{aligned}
|f(x, 0)| & \leq \alpha_{1}^{-1}(V(f(x, 0))) \leq \alpha_{1}^{-1}(V(x)-\bar{\alpha}(|x|)) \\
& \leq \alpha_{1}^{-1}\left(\alpha_{2}(|x|)-\bar{\alpha}(|x|)\right)
\end{aligned}
$$

Since by (7) $\bar{\alpha}(|x|) \leq V(x) \leq \alpha_{2}(|x|)$ for all $x \in \mathbb{R}^{n}$, with equality if and only if $x=0$, the function $\alpha_{1}^{-1}\left(\alpha_{2}(s)-\bar{\alpha}(s)\right)$ is positive definite. Define $\bar{\gamma} \in \mathcal{K}_{\infty}$ by $\bar{\gamma}(s) \doteq \max \left\{s, \alpha_{1}^{-1}\left(\alpha_{2}(s)-\bar{\alpha}(s)\right)\right\}$ for all $s \in \mathbb{R}_{\geq 0}$ so that $|f(x, 0)| \leq \bar{\gamma}(|x|)$ for all $x \in \mathbb{R}^{n}$.

Now, if for all $r>0$ we define

$$
\hat{\gamma}(r) \doteq \sup \{|f(x, w)-f(x, 0)|:|x| \leq r,|w| \leq r\}
$$

then for all $r_{1} \geq r$ we obtain $\hat{\gamma}(r) \leq \gamma_{r_{1}}(r)$ which implies $\hat{\gamma}(r) \rightarrow 0$ as $r \rightarrow 0$. Moreover, $\hat{\gamma}(r)$ is finite for all $r>0$. Hence, we may overbound $\hat{\gamma}$ with a function $\gamma \in \mathcal{K}_{\infty}$.

It is now sufficient to show that there exists $\varphi \in \mathcal{K}$ such that the implication in (12) holds. To this end, let $|x|<\chi(|w|)$. Then

$$
\begin{aligned}
|f(x, w)| & =|f(x, w)-f(x, 0)+f(x, 0)| \\
& \leq \gamma(\max \{|w|, \chi(|w|)\})+\bar{\gamma}(|x|) \\
& \leq \gamma(\max \{|w|, \chi(|w|)\})+\bar{\gamma}(\chi(|w|))=: \tilde{\gamma}(|w|)
\end{aligned}
$$

implying $V(f(x, w)) \leq \alpha_{2}(|f(x, w)|) \leq \alpha_{2}(\tilde{\gamma}(|w|))$. This shows the desired inequality with $\varphi(r) \doteq \alpha_{2}(\tilde{\gamma}(|w|))$.

We note that the map $f(x, w)=\nu(w) \kappa(x)$ in (9) of Example 3.3 does not satisfy the required continuity property of Proposition 4.6. To see this, we first observe that $|f(x, w)-f(x, 0)|=$ 
$|f(x, w)|$. Choose $r=1$ and any $\gamma_{1} \in \mathcal{K}_{\infty}$. Then, with $w=1$, we see that

$$
|f(x, 1)|=\frac{1}{|x|}, \quad \forall x \in(-1,1) \backslash\{0\}
$$

so that $|f(x, 1)|>\gamma_{1}(1)$ for some $x \in(0,1)$.

Using the strong implication-form ISS-Lyapunov function allows us to prove a variant of Proposition 2.5.

Proposition 4.7: Suppose $V: \mathbb{R}^{n} \rightarrow \mathbb{R}_{\geq 0}, \alpha_{1}, \alpha_{2} \in \mathcal{K}_{\infty}$ and $\alpha, \sigma \in \mathcal{K}$ satisfy (3) and (4). If there exists $\rho \in \mathcal{P}$ such that $\alpha(s)=\rho(s)+\sigma(s)$ for all $s \in \mathbb{R}_{\geq 0}$ then $V$ satisfies (11) and (12) with $\chi \doteq \mathrm{id}, \hat{\rho} \doteq \rho$, and $\hat{\varphi} \doteq \gamma+\sigma$ where $\gamma \in \mathcal{K}$ is such that $\gamma>\alpha_{2}-\alpha$, and hence (1) is ISS.

Proof: By assumption, we have $V(f(x, w))-V(x) \leq-\rho(|x|)-\sigma(|x|)+\sigma(|w|)$. Therefore $|x| \geq|w|$ implies $V(f(x, w))-V(x) \leq-\rho(|x|)$ and $|x|<|w|$ implies

$$
\begin{aligned}
V(f(x, w)) & \leq V(x)-\alpha(|x|)+\sigma(|w|) \\
& \leq \alpha_{2}(|x|)-\alpha(|x|)+\sigma(|w|) \leq \gamma(|w|)+\sigma(|w|) .
\end{aligned}
$$

Thus, $V$ is a strong implication-form ISS-Lyapunov function and by Theorem 4.4 the system is ISS.

We note that the assumptions of Propositions 2.5 and 4.7 do not imply each other. Clearly, $\sup \alpha>\sup \sigma$ does not imply $\alpha(r)>\sigma(r)$ for all $r>0$. Conversely, one checks that the functions $\sigma(s)=s /(1+s)$ and $\alpha(s)=\sigma(s)+\rho(s)$ with $\rho(s)=\min \{s, 1 /(2+2 s)\}$ satisfy the assumption of Proposition 4.7 although $\sup \alpha=1=\sup \sigma$. From a quantitative point of view, Proposition 4.7 provides a stronger statement than Proposition 2.5, as it maintains the functions of the given ISS-Lyapunov function without requiring a rescaling.

\section{CONCLusions}

In this paper we have provided a complete characterization of ISS-Lyapunov functions for discrete-time systems with discontinuous dynamics. In contrast to the original definition of an ISS-Lyapunov function in [23], we here observed that an implication-form ISS-Lyapunov function does not necessarily imply ISS of the system (1). In order to counter this difficulty, we proposed an alternative strong implication-form ISS-Lyapunov function and demonstrated that this ISS-Lyapunov function satisfies many of the desirable properties that hold in a more classical setting such as equivalence to an ISS-Lyapunov function in dissipative form and that 
this strong implication-form ISS-Lyapunov function is both necessary and sufficient for ISS of discrete-time systems with discontinuous dynamics.

In addition, we have presented results on the nonlinear scaling of such strong implicationform ISS-Lyapunov functions and have demonstrated that it is always possible to move between decrease rates that are given by positive definite functions, functions of class- $\mathcal{K}_{\infty}$, or even an exponential decrease. In all cases, we have explicitly shown how these various functions are related to each other in a quantitative manner.

\section{PROOFS}

In what follows, when it improves readability and causes no confusion, we drop the arguments for ISS-Lyapunov functions and simply write $V^{+} \doteq V(f(x, w))$ and $V \doteq V(x)$.

\section{A. Proof of “(i) $\Rightarrow$ (iii)” in Theorem 2.3}

Our proof relies on a converse Lyapunov theorem for difference inclusions. We denote the set of solutions to the difference inclusion

$$
x^{+} \in F(x), \quad x \in \mathbb{R}^{n}
$$

defined by the set-valued mapping $F: \mathbb{R}^{n} \rightrightarrows \mathbb{R}^{n}$ and from an initial condition $x \in \mathbb{R}^{n}$ by $\mathcal{S}(x)$. A solution $\phi \in \mathcal{S}(x)$ is a function $\phi: \mathbb{Z}_{\geq 0} \times \mathbb{R}^{n} \rightarrow \mathbb{R}^{n}$ such that $\phi(0, x)=x$ and $\phi(k+1, x) \in F(\phi(k, x))$ for all $k \in \mathbb{Z}_{\geq 0}$.

Definition 6.1: The difference inclusion (14) is said to be $\mathcal{K} \mathcal{L}$-stable if there exists $\beta \in \mathcal{K} \mathcal{L}$ so that

$$
|\phi(k, x)| \leq \beta(|x|, k), \quad \forall x \in \mathbb{R}^{n}, \phi \in \mathcal{S}(x), k \in \mathbb{Z}_{\geq 0}
$$

Theorem 6.2: If the difference inclusion (14) is $\mathcal{K} \mathcal{L}$-stable then, for any given $\lambda \in(0,1)$ there exists an exponential-decrease Lyapunov function; i.e., there exist functions $V: \mathbb{R}^{n} \rightarrow \mathbb{R}_{\geq 0}$ and $\alpha_{1}, \alpha_{2} \in \mathcal{K}_{\infty}$ so that

$$
\begin{gathered}
\alpha_{1}(|x|) \leq V(x) \leq \alpha_{2}(|x|) \\
V(\phi(1, x)) \leq \lambda V(x)
\end{gathered}
$$

for all $x \in \mathbb{R}^{n}$ and $\phi(1, x) \in F(x)$. 
Proof: The proof follows that of [12, Theorem 2.7] where, here, we need not worry about regularity of the Lyapunov function.

Given $\beta \in \mathcal{K} \mathcal{L}$ and $\lambda \in(0,1)$, Sontag's lemma on $\mathcal{K} \mathcal{L}$-estimates [21, Proposition 7] yields $\alpha_{1}, \alpha_{2} \in \mathcal{K}_{\infty}$ so that

$$
\alpha_{1}(\beta(s, k)) \leq \alpha_{2}(s) \lambda^{k}, \quad \forall s \in \mathbb{R}_{\geq 0}, \quad k \in \mathbb{Z}_{\geq 0} .
$$

Define $V(x) \doteq \sup _{k \in \mathbb{Z}_{\geq 0}} \sup _{\phi \in \mathcal{S}(x)} \alpha_{1}(|\phi(k, x)|) \lambda^{-k}$ for all $x \in \mathbb{R}^{n}$. Then, for all $x \in \mathbb{R}^{n}$, $V(x) \geq \sup _{\phi \in \mathcal{S}(x)} \alpha_{1}(|\phi(0, x)|) \lambda^{0}=\alpha_{1}(|x|)$ and

$$
V(x) \leq \sup _{k \in \mathbb{Z}_{\geq 0}} \alpha_{1}(\beta(|x|, k)) \lambda^{-k} \leq \sup _{k \in \mathbb{Z}_{\geq 0}} \alpha_{2}(|x|) \lambda^{k} \lambda^{-k}=\alpha_{2}(|x|)
$$

so that $V(x)$ satisfies the desired upper and lower bounds (16). The desired decrease condition follows as

$$
\begin{aligned}
V(\phi(1, x)) & =\sup _{k \in \mathbb{Z}_{\geq 0}} \sup _{\psi \in \mathcal{S}(\phi(1, x))} \alpha_{1}(|\psi(k, \phi(1, x))|) \lambda^{-k} \\
& \leq \sup _{k \in \mathbb{Z} \geq 1} \sup _{\phi \in \mathcal{S}(x)} \alpha_{1}(|\phi(k, x)|) \lambda^{-k+1} \\
& \leq \sup _{k \in \mathbb{Z} \geq 0} \sup _{\phi \in \mathcal{S}(x)} \alpha_{1}(|\phi(k, x)|) \lambda^{-k+1}=\lambda V(x)
\end{aligned}
$$

for all $x \in \mathbb{R}^{n}$.

In order to demonstrate that ISS implies the existence of an ISS-Lyapunov function, we follow the standard argument as in [23] and [7]. Denote the closed unit ball in $\mathbb{R}^{m}$ by $\mathcal{B}^{m}$. We show that there exists a $\mu \in \mathcal{K}_{\infty}$ such that the differential inclusion defined by

$$
x(k+1) \in f\left(x(k), \mu(|x(k)|) \mathcal{B}^{m}\right)
$$

is $\mathcal{K} \mathcal{L}$-stable, allowing us to appeal to Theorem 6.2 to obtain an ISS-Lyapunov function. We denote the solution set of (19) from an initial condition $x \in \mathbb{R}^{n}$ by $\mathcal{S}_{\mu}(x)$.

Proposition 6.3: [12, Proposition 2.2.] The difference inclusion (14) is $\mathcal{K} \mathcal{L}$-stable if and only if the following hold:

1) (Uniform stability): There exists $\gamma \in \mathcal{K}_{\infty}$ so that, for each $x \in \mathbb{R}^{n}$, all solutions $\phi \in \mathcal{S}(x)$ satisfy

$$
|\phi(k, x)| \leq \gamma(|x|), \quad \forall k \in \mathbb{Z}_{\geq 0}
$$


2) (Uniform global attractivity): For each $r, \varepsilon \in \mathbb{R}_{>0}$, there exists $K(r, \varepsilon)>0$ so that, for each $x \in \mathbb{R}^{n}$, all solutions $\phi \in \mathcal{S}(x)$ satisfy

$$
|x| \leq r, \quad k \in \mathbb{Z}_{\geq K(r, \varepsilon)} \quad \Rightarrow \quad|\phi(k, x)| \leq \varepsilon .
$$

Lemma 6.4: If (1) is ISS then there exists $\mu \in \mathcal{K}_{\infty}$ such that the difference inclusion (19) is $\mathcal{K} \mathcal{L}$-stable.

Proof: Without loss of generality, we assume that $\gamma \in \mathcal{K}$ from (2) satisfies $\gamma(r) \geq r$. For all $s \in \mathbb{R}_{\geq 0}$, define $\alpha, \mu \in \mathcal{K}_{\infty}$ as

$$
\alpha(s) \doteq \max \left\{\gamma(\beta(s, 0)), \gamma\left(\frac{1}{2} s\right)\right\}, \quad \mu(s) \doteq \frac{1}{2} \gamma^{-1}\left(\frac{1}{4} \alpha^{-1}(s)\right) .
$$

Claim 6.5: For any $x \in \mathbb{R}^{n}$ and $\phi \in \mathcal{S}_{\mu}(x)$ we have

$$
\gamma \circ \mu(|\phi(k, x)|) \leq \frac{1}{2}|x|, \quad \forall k \in \mathbb{Z}_{\geq 0} .
$$

Proof: The definition of $\alpha$ implies $|x| \leq \beta(|x|, 0) \leq \alpha(|x|)$ so that

$$
\gamma \circ \mu(|x|) \leq \frac{1}{4} \alpha^{-1}(|x|) \leq \frac{1}{4}|x| .
$$

Let $k_{1} \doteq \min \left\{k \in \mathbb{Z}_{\geq 0}: \gamma \circ \mu(|\phi(k, x)|)>\frac{1}{2}|x|\right\}$ and note that (21) implies $k_{1} \in \mathbb{Z}_{\geq 1}$. In order to obtain a contradiction, assume $k_{1}<\infty$. Then (20) holds for all $k \in \mathbb{Z}_{\left[0, k_{1}-1\right]}$. Therefore, $\gamma\left(\left|\mu(|\phi(k, x)|) \mathcal{B}^{m}\right|\right) \leq \frac{1}{2}|x|$ for all $\phi \in \mathcal{S}_{\mu}(x)$ and $k \in \mathbb{Z}_{\left[0, k_{1}-1\right]}$. Applying $\gamma \in \mathcal{K}_{\infty}$ to both sides of the ISS-estimate (2) in conjunction with this fact yields

$$
\gamma(|\phi(k, x)|) \leq \max \left\{\gamma(\beta(|x|, 0)), \gamma\left(\frac{1}{2}|x|\right)\right\}=\alpha(|x|),
$$

for all $\phi \in \mathcal{S}_{\mu}(x), k \in \mathbb{Z}_{\left[0, k_{1}-1\right]}$. Then, using the definition of $\mu$, the ISS-estimate (2), and (22), we have

$$
\begin{aligned}
& \gamma \circ \mu\left(\left|\phi\left(k_{1}, x\right)\right|\right) \leq \frac{1}{4} \alpha^{-1}\left(\left|\phi\left(k_{1}, x\right)\right|\right) \\
& \leq \frac{1}{4} \max \left\{\alpha^{-1}(\beta(|x|, k)) \max _{j \in \mathbb{Z}_{\left[0, k_{1}-1\right]}} \alpha^{-1} \circ \gamma(|\phi(j, x)|)\right\} \\
& \leq \frac{1}{4} \max \{|x|,|x|\}=\frac{1}{4}|x|
\end{aligned}
$$

which contradicts the definition of $k_{1}$ and hence proves the claim.

We now prove $\mathcal{K} \mathcal{L}$-stability of difference inclusion (19) by proving uniform stability and uniform global attractivity and then appeal to Proposition 6.3. 
Uniform stability follows from (2), (20), and $\gamma(s) \geq s$ as

$$
\begin{aligned}
|\phi(k, x)| & \leq \max \left\{\beta(|x|, k), \max _{i \in \mathbb{Z}_{[0, k-1]}} \gamma\left(\mid \mu(|\phi(i, x)|) \mathcal{B}^{m}\right)\right\} \\
& \leq \max \left\{\beta(|x|, k), \frac{1}{2}|x|\right\} \leq \alpha(|x|) .
\end{aligned}
$$

To establish uniform global attractivity, we use (23). Since $\beta \in \mathcal{K} \mathcal{L}$, for each $r \in \mathbb{R}_{\geq 0}$ there

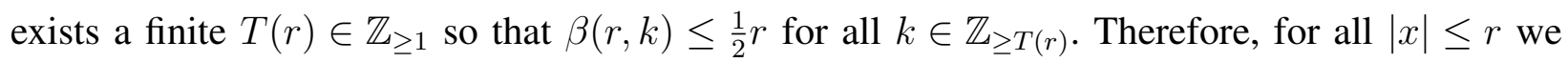
have $|\phi(k, x)| \leq \frac{1}{2} r$ for all $\phi \in \mathcal{S}_{\mu}(x)$ and $k \in \mathbb{Z}_{\geq T(r)}$.

Fix any $\varepsilon \in \mathbb{R}_{>0}$ and let $k \in \mathbb{Z}_{\geq 1}$ be such that $2^{-k} r \leq \varepsilon$. Define $r_{1} \doteq r, r_{i} \doteq \frac{1}{2} r_{i-1}$ for all $i \in \mathbb{Z}_{\geq 2}$, and $K(r, \varepsilon) \doteq \sum_{i=1}^{k} T\left(r_{i}\right)$. Then $|\phi(k, x)| \leq 2^{-k} r \leq \varepsilon$ for all $|x| \leq r, \phi \in \mathcal{S}_{\mu}(x)$, and $k \in \mathbb{Z}_{\geq K(r, \varepsilon)}$. Therefore, the difference inclusion (19) is $\mathcal{K} \mathcal{L}$-stable.

We now complete the proof of "(i) $\Rightarrow$ (iii)" in Theorem 2.3. Since (1) is ISS, the difference inclusion (19) is $\mathcal{K} \mathcal{L}$-stable, and by Theorem 6.2 , for any $\lambda \in(0,1)$, there exist functions $V: \mathbb{R}^{n} \rightarrow \mathbb{R}_{\geq 0}$ and $\alpha_{1}, \alpha_{2} \in \mathcal{K}_{\infty}$ so that (16) and (17) hold for the difference inclusion given by (19). This then implies that

$$
|w| \leq \mu(|x|) \quad \Rightarrow \quad V(\phi(1, x, w)) \leq \lambda V(x)
$$

for all $x \in \mathbb{R}^{n}, w \in \mathbb{R}^{m}$, and $\phi(1, x, w)=f(x, w)$.

It remains to show that the function $V$ satisfies (5). Let $\beta \in \mathcal{K} \mathcal{L}$ and $\gamma \in \mathcal{K}_{\infty}$ come from the ISS-estimate (2) and $\alpha_{2} \in \mathcal{K}_{\infty}$ the upper bound in (16). Define $\sigma \in \mathcal{K}$ by $\sigma(s) \doteq$ $\alpha_{2}\left(\beta\left(\mu^{-1}(s), 1\right)+\gamma(s)\right)$ for all $s \in \mathbb{R}_{\geq 0}$. For $|w|>\mu(|x|)$ we have

$$
\begin{aligned}
V(f(x, w)) & \leq \alpha_{2}(|f(x, w)|) \leq \alpha_{2}(\beta(|x|, 1)+\gamma(|w|)) \\
& \leq \alpha_{2}\left(\beta\left(\mu^{-1}(|w|), 1\right)+\gamma(|w|)\right)=\sigma(|w|) .
\end{aligned}
$$

Together with (24) we then have $V(f(x, w)) \leq \lambda V(x)+\sigma(|w|)$ for all $x \in \mathbb{R}^{n}$ and $w \in \mathbb{R}^{m}$.

\section{B. Proof of Proposition 2.5}

The proof closely follows [17, Lemma 1]. The condition $\sup \alpha>\sup \sigma$ implies there exists $c>1$ so that $\sup \alpha>c \sup \sigma$. 
Define $\varphi \in \mathcal{K}_{\infty}$ by $\varphi(s) \doteq \int_{0}^{s} \alpha(r) d r$ for all $s \in \mathbb{R}_{\geq 0}$ and $\widehat{V} \doteq \varphi(V)$. For $V^{+} \leq \frac{V}{2}$ we have $\varphi\left(V^{+}\right)-\varphi(V) \leq \varphi\left(\frac{V}{2}\right)-\varphi(V)$

$$
\leq \alpha\left(\frac{V}{2}\right)\left(\frac{V}{2}-V\right) \leq-\alpha\left(\frac{V}{2}\right) \frac{V}{2}+\sigma(|w|) .
$$

For $V^{+}>\frac{V}{2}$, the mean value theorem yields $\varphi\left(V^{+}\right)-\varphi(V)=\alpha\left(V^{+}-\theta\left(V^{+}-V\right)\right)\left(V^{+}-V\right)$ for some $\theta \in(0,1)$ and consequently

$$
\varphi\left(V^{+}\right)-\varphi(V) \leq \alpha\left(V^{+}\right)\left(V^{+}-V\right) .
$$

For $V^{+}>\frac{V}{2}$ consider two cases. The first is when $\frac{1}{c} \alpha(V)>\sigma(|w|)$. Then

$$
\begin{aligned}
& \alpha\left(V^{+}\right)\left(V^{+}-V\right) \leq \alpha\left(V^{+}\right)\left(-\alpha(V)+\frac{1}{c} \alpha(V)\right) \\
& \leq-\alpha\left(\frac{V}{2}\right)\left(1-\frac{1}{c}\right) \alpha(V) \leq-\alpha\left(\frac{V}{2}\right)\left(1-\frac{1}{c}\right) \alpha(V)+\sigma(|w|) .
\end{aligned}
$$

The second is $V^{+}>\frac{V}{2}$ and $\frac{1}{c} \alpha(V) \leq \sigma(|w|)$. Since $c \sup \sigma<\sup \alpha$, we see that $\alpha^{-1}(s)$ exists for all $s \in[0, c \sup \sigma]$ and hence we have that $\alpha\left(V^{+}\right) \leq \alpha(V+\sigma(|w|)) \leq \alpha\left(\alpha^{-1}(c \sigma(|w|))+\sigma(|w|)\right)$.

Define $\gamma(s) \doteq \alpha\left(\alpha^{-1}(c \sigma(s))+\sigma(s)\right)$. Then

$$
\begin{aligned}
& \alpha\left(V^{+}\right)\left(V^{+}-V\right) \leq \alpha\left(V^{+}\right)(-\alpha(V)+\sigma(|w|)) \\
& \leq-\alpha\left(V^{+}\right) \alpha(V)+\gamma(|w|) \sigma(|w|) \\
& \leq-\alpha\left(\frac{V}{2}\right) \alpha(V)+\gamma(|w|) \sigma(|w|) .
\end{aligned}
$$

Therefore, with $\bar{\alpha} \in \mathcal{K}_{\infty}$ and $\bar{\sigma} \in \mathcal{K}$ defined by

$$
\begin{aligned}
& \bar{\alpha}(s) \doteq \min \left\{\alpha\left(\frac{s}{2}\right) \frac{s}{2}, \alpha\left(\frac{s}{2}\right)\left(1-\frac{1}{c}\right) \alpha(s), \alpha\left(\frac{s}{2}\right) \alpha(s)\right\} \\
& \bar{\sigma}(s) \doteq \max \{\sigma(s), \gamma(s) \sigma(s)\}
\end{aligned}
$$

combining (25)-(28) yields $\widehat{V}^{+}-\widehat{V} \leq-\bar{\alpha}(V)+\bar{\sigma}(|w|) \leq-\bar{\alpha} \circ \alpha_{1}(|x|)+\bar{\sigma}(|w|)$ so that (1) is ISS.

\section{Proof of Proposition 3.2 and Remark 4.3}

Following Remark 4.3 we prove Proposition 3.2 both for the weak and for the strong implicationform. We include the necessary computations for (12) in remarks in what follows. We first observe that the implications (iii) $\Rightarrow$ (ii) $\Rightarrow$ (i) are trivial. 
1) Positive Definite to $\mathcal{K}_{\infty}:(i) \Rightarrow(i i)$ : We start from an ISS-Lyapunov function with a positive definite decrease rate; i.e., $V: \mathbb{R}^{n} \rightarrow \mathbb{R}_{\geq 0}, \alpha_{1}, \alpha_{2} \in \mathcal{K}_{\infty}, \chi \in \mathcal{K}, \rho \in \mathcal{P}$ satisfying (3) and (6).

For $\rho \in \mathcal{P}$, [1, Lemma IV.1] ([9, Lemma 12]) yields $\kappa \in \mathcal{K}_{\infty}$ and $\sigma \in \mathcal{L}$ so that $\rho(s) \geq$ $\kappa(s) \sigma(s)$ for all $s \in \mathbb{R}_{\geq 0}$. Using (3) we see that, for all $x \in \mathbb{R}^{n},|x| \geq \chi(|w|)$ implies

$$
\begin{aligned}
V(f(x, w))-V(x) \leq-\rho(|x|) \leq & -\kappa(|x|) \sigma(|x|) \\
& \leq-\left(\kappa \circ \alpha_{2}^{-1}(V(x))\right)\left(\sigma \circ \alpha_{1}^{-1}(V(x))\right)=-\hat{\rho}(V(x))
\end{aligned}
$$

where $\hat{\rho}(s) \doteq\left(\kappa \circ \alpha_{2}^{-1}(s)\right)\left(\sigma \circ \alpha_{1}^{-1}(s)\right)$ for all $s \in \mathbb{R}_{\geq 0}$ is positive definite.

From here, we follow [8, Lemma 2.8]. Let $\bar{\kappa} \in \mathcal{K}_{\infty}$ be such that

$$
\bar{\kappa}\left(\frac{s}{2}\right) \hat{\rho}(s) \geq s, \quad \forall s \geq 1
$$

and define $\hat{\alpha} \in \mathcal{K}_{\infty}$ by

$$
\hat{\alpha}(s) \doteq s+\int_{0}^{s} \bar{\kappa}(r) d r, \quad \text { so that } \quad \hat{\alpha}^{\prime}(s)=1+\bar{\kappa}(s)
$$

for all $s \in \mathbb{R}_{>0}$. Therefore, $\hat{\alpha}^{\prime}$ is strictly increasing.

Define $\widehat{V}(x) \doteq \hat{\alpha}(V(x))$ for all $x \in \mathbb{R}^{n}$ and observe that with the $\mathcal{K}_{\infty}$ functions $\hat{\alpha}_{1} \doteq \hat{\alpha} \circ \alpha_{1}$ and $\hat{\alpha}_{2} \doteq \hat{\alpha} \circ \alpha_{2}$ we have $\hat{\alpha}_{1}(|x|) \leq \widehat{V}(x) \leq \hat{\alpha}_{2}(|x|)$.

Remark 6.6: In order to prove that (12) is maintained under this scaling, let $\hat{\varphi} \in \mathcal{K}$ be given by $\hat{\varphi} \doteq \hat{\alpha} \circ \varphi$ so that (12) implies, for $|x|<\chi(|w|), \widehat{V}^{+}=\hat{\alpha}\left(V^{+}\right) \leq \hat{\alpha} \circ \varphi(|w|)=\hat{\varphi}(|w|)$.

In what follows we assume $|x| \geq \chi(|w|)$. Since $\hat{\alpha}$ is differentiable, the mean value theorem yields the existence of $\theta \in(0,1)$ so that

$$
\hat{\alpha}\left(V^{+}\right)-\hat{\alpha}(V)=\hat{\alpha}^{\prime}\left(V^{+}+\theta\left(V-V^{+}\right)\right)\left(V^{+}-V\right) .
$$

Note that, as a consequence of (29), $V^{+}-V \leq 0$.

We first restrict attention to $V \geq 1$ and consider two cases. First, we assume $V^{+} \leq \frac{V}{2}$ and note that $\hat{\alpha}^{\prime}(s) \geq 1$ for all $s \in \mathbb{R}_{\geq 0}$. Then

$$
\widehat{V}^{+}-\widehat{V} \leq V^{+}-V \leq-\frac{V}{2} .
$$

Now suppose $V^{+} \geq \frac{V}{2}$. In this case, using $V-V^{+} \geq 0$ and (31), we have $\hat{\alpha}^{\prime}\left(V^{+}+\theta(V-\right.$ $\left.\left.V^{+}\right)\right) \geq \hat{\alpha}^{\prime}\left(V^{+}\right) \geq \hat{\alpha}^{\prime}\left(\frac{V}{2}\right)>\bar{\kappa}\left(\frac{V}{2}\right)$. 
Combining this with (29) and (30), for $V \geq 1$ we obtain

$$
\widehat{V}^{+}-\widehat{V} \leq \bar{\kappa}\left(\frac{V}{2}\right)\left(V^{+}-V\right) \leq-\bar{\kappa}\left(\frac{V}{2}\right) \hat{\rho}(V) \leq-V
$$

Combining (33) and (34) we see that, for $V \geq 1, \widehat{V}^{+}-\widehat{V} \leq-\frac{V}{2}$ holds. For $V \leq 1$, we note that by definition (31) and (29) we have

$$
\begin{aligned}
\widehat{V}^{+}-\widehat{V} & =V^{+}+\int_{0}^{V^{+}} \bar{\kappa}(r) d r-V-\int_{0}^{V} \bar{\kappa}(r) d r \\
& \leq V^{+}-V \leq-\hat{\rho}(V) .
\end{aligned}
$$

Take $\check{\alpha} \in \mathcal{K}_{\infty}$ so that $\check{\alpha}(s) \leq \hat{\rho}(s)$ for all $s \in[0,1]$ and $\check{\alpha}(s) \leq \frac{s}{2}$ for all $s \geq 1$. Finally, let $\bar{\alpha} \in \mathcal{K}_{\infty}$ be defined as $\bar{\alpha} \doteq \check{\alpha} \circ \alpha_{1}$ so that $|x| \geq \chi(|w|)$ implies

$$
\widehat{V}(f(x, w))-\widehat{V}(x) \leq-\check{\alpha}(V(x)) \leq-\check{\alpha} \circ \alpha_{1}(|x|)=-\bar{\alpha}(|x|) .
$$

2) $\mathcal{K}_{\infty}$ to Exponential: $($ ii $) \Rightarrow($ iii $)$ : Define $\mu \in \mathcal{K}_{\infty}$ by $\mu(s) \doteq \min \left\{\bar{\alpha} \circ \hat{\alpha}_{2}^{-1}(s), \frac{s}{2}\right\}$ and note that id $-\mu \in \mathcal{K}_{\infty}$. Therefore, $|x| \geq \chi(|w|)$ implies $\widehat{V}^{+} \leq \widehat{V}-\mu(\widehat{V})=(\mathrm{id}-\mu)(\widehat{V})$.

Select any $\hat{\lambda} \in(0,1)$. Then $\left[9\right.$, Corollary 1] yields $\tilde{\alpha} \in \mathcal{K}_{\infty}$ so that $\tilde{\alpha} \circ(\operatorname{id}-\mu)(s)=\hat{\lambda} \hat{\mu}(s)$ for all $s \in \mathbb{R}_{\geq 0}$.

Define $\widetilde{V} \doteq \tilde{\alpha}(\widehat{V})$ and note that, with $\mathcal{K}_{\infty}$ functions $\tilde{\alpha}_{1} \doteq \tilde{\alpha} \circ \hat{\alpha}_{1}$ and $\tilde{\alpha}_{2} \doteq \tilde{\alpha} \circ \hat{\alpha}_{2}$ the inequality $\tilde{\alpha}_{1}(|x|) \leq \widetilde{V}(x) \leq \tilde{\alpha}_{2}(|x|)$ holds. Furthermore, $|x| \geq \chi(|w|)$ implies

$$
\widetilde{V}^{+}=\tilde{\alpha}\left(\widehat{V}^{+}\right) \leq \tilde{\alpha} \circ(\mathrm{id}-\mu)(\widehat{V})=\hat{\lambda} \tilde{\alpha}(\widehat{V})=\hat{\lambda} \widetilde{V}
$$

Remark 6.7: To additionally demonstrate that (12) persists, take $\hat{\varphi}$ from Remark 6.6 and define $\tilde{\varphi} \doteq \tilde{\alpha} \circ \hat{\varphi} \in \mathcal{K}$ so that, for $|x|<\chi(|w|)$ we obtain $\widetilde{V}^{+}=\tilde{\alpha}\left(\widehat{V}^{+}\right) \leq \tilde{\alpha} \circ \hat{\varphi}(|w|)=\tilde{\varphi}(|w|)$.

\section{ACKNOWLEDGEMENT}

The authors are grateful to Rob Gielen for helpful discussions on the result of Theorem 2.3.

\section{REFERENCES}

[1] D. Angeli, E. D. Sontag, and Y. Wang. A characterization of integral input-to-state stability. IEEE Transactions on Automatic Control, 45(6):1082-1097, 2000.

[2] K. Aström. Event based control. In A. Astolfi and L. Marconi, editors, Analysis and Design of Nonlinear Control Systems, pages 127-147, Berlin, 2008. Springer-Verlag.

[3] G. Grimm, M. J. Messina, S. E. Tuna, and A. R. Teel. Examples when nonlinear model predictive control is nonrobust. Automatica, 40:1729-1738, 2004. 
[4] L. Grüne and F. Müller. Global optimal control of quantized systems. In Proceedings of the 18th International Symposium on Mathematical Theory of Networks and Systems, pages 1231-1237, Budapest, Hungary, 2010.

[5] L. Grüne and J. Pannek. Nonlinear Model Predictive Control. Theory and Algorithms. Springer-Verlag, London, 2011.

[6] L. Grüne and M. Sigurani. Numerical ISS controller design via a dynamic game approach. In Proceedings of the 52nd IEEE Conference on Decision and Control, pages 1732-1737, Florence, Italy, 2013.

[7] Z.-P. Jiang and Y. Wang. Input-to-state stability for discrete-time nonlinear systems. Automatica, 37:857-869, 2001.

[8] Z.-P. Jiang and Y. Wang. A converse Lyapunov theorem for discrete-time systems with disturbances. Systems and Control Letters, 45:49-58, 2002.

[9] C. M. Kellett. A compendium of comparsion function results. Mathematics of Controls, Signals and Systems, 2014. DOI: 10.1007/s00498-014-0128-8.

[10] C. M. Kellett and A. R. Teel. Discrete-time asymptotic controllability implies smooth control-Lyapunov function. Systems and Control Letters, 52(5):349-359, August 2004.

[11] C. M. Kellett and A. R. Teel. Smooth Lyapunov functions and robustness of stability for difference inclusions. Systems and Control Letters, 52(5):395-405, August 2004.

[12] C. M. Kellett and A. R. Teel. On the robustness of $\mathcal{K} \mathcal{L}$-stability for difference inclusions: Smooth discrete-time Lyapunov functions. SIAM Journal on Control and Optimization, 44(3):777-800, 2005.

[13] M. Lazar, W. Heemels, and A. R. Teel. Lyapunov functions, stability and input-to-state stability subtleties for discrete-time discontinuous systems. IEEE Transactions on Automatic Control, 54(10):2421-2425, 2009.

[14] J. Lunze and D. Lehmann. A state-feedback approach to event-based control. Automatica, 46(1):211-215, 2010.

[15] L. Magni, D. M. Raimondo, and R. Scattolini. Regional input-to-state stability for nonlinear model predictive control. IEEE Transactions on Automatic Control, 51(9):1548-1553, 2006.

[16] D. Nešić and D. Liberzon. A unified framework for design and analysis of networked and quantized control systems. IEEE Trans. Automat. Control, 54(4):732-747, 2009.

[17] D. Nešić and A. R. Teel. Changing supply functions in input to state stable systems: The discrete-time case. IEEE Transactions on Automatic Control, 46(6):960-962, June 2001.

[18] B. Picasso, D. Desiderio, and R. Scattolini. Robust stability analysis of nonlinear discrete-time systems with application to MPC. IEEE Transactions on Automatic Control, 57(1):185-191, January 2012.

[19] L. Praly and Y. Wang. Stabilization in spite of matched unmodeled dynamics and an equivalent definition of input-to-state stability. Mathematics of Control, Signals, and Systems, 9:1-33, 1996.

[20] E. D. Sontag. Smooth stabilization implies coprime factorization. IEEE Transactions on Automatic Control, 34(4):435-443, April 1989.

[21] E. D. Sontag. Comments on integral variants of ISS. Systems and Control Letters, 34(1-2):93-100, 1998.

[22] E. D. Sontag and A. R. Teel. Changing supply functions in input/state stable systems. IEEE Transactions on Automatic Control, 40(8):1476-1478, 1995.

[23] E. D. Sontag and Y. Wang. On characterizations of the input-to-state stability property. Systems and Control Letters, 24:351-359, 1995. 